\title{
La fragmentación educativa en Argentina: debates recientes
}

\section{Fragmentação educacional na Argentina: debates recentes}

\section{Educational fragmentation in Argentina: recent debates}

\author{
Andrés Santos Sharpe \\ Universidad de Buenos Aires (UBA), Cidade Autônoma de Buenos Aires - Argentina \\ Consejo Nacional de Investigaciones Científicas y Técnicas (Conicet), Cidade Autônoma de Buenos \\ Aires - Argentina
}

\begin{abstract}
Resumen
El artículo presenta debates académicos recientes publicados en revistas científicas en torno a la fragmentación educativa, particularizando en el caso argentino. Para ello, en primer lugar, se recuperan los análisis históricos en torno a la desigualdad educativa en el país, con foco aquellos elementos que permitieron la emergencia de los estudios sobre la fragmentación educativa y sus especificidades en relación al sistema educativo argentino. La segunda parte del artículo presenta los distintos modos en que se expresa la fragmentación educativa para el caso argentino en la actualidad a la vez que nos preguntamos por sus consecuencias por fuera del ámbito estrictamente escolar. Resumidamente, el artículo gira en torno a la pregunta sobre cuáles son las miradas históricas y las formas de procesar la desigualdad que permitieron la elaboración del concepto de fragmentación.
\end{abstract}

Palabras clave: Fragmentación, Sistema educativo argentino, Desigualdad educativa

\section{Resumo}

Este artigo apresenta debates acadêmicos recentes publicados em revistas científicas em torno do conceito de fragmentação educativa, particularmente, no caso argentino. Para isso, em primeiro lugar, recuperam-se as análises históricas em torno da desigualdade educacional no país, enfatizando aqueles elementos que permitiram o surgimento de estudos sobre a fragmentação educacional e suas especificidades em relação ao sistema educacional argentino. A segunda parte do artigo apresenta as diferentes formas de a fragmentação educacional se expressar no caso argentino na atualidade, enquanto nos questionamos sobre suas consequências fora do ambiente estritamente escolar. Em suma, o artigo gira em torno da questão de quais são as visões históricas e as formas de tratamento da desigualdade que permitiram o desenvolvimento do conceito de fragmentação.

Palavras-chave: Fragmentação, Sistema educacional argentino, desigualdade educativa

\begin{abstract}
This paper presents recent academic debates published in scientific journals on educational fragmentation, focusing on the Argentine case. First, it retrieves a historical analysis of educational inequality in Argentina, what elements allowed the emergence of studies on educational fragmentation, and what specificities they found in the Argentine educational system. The second part of the article presents the different ways in which educational fragmentation is expressed Argentina while wondering about its consequences outside the strictly school environment. In short, the article is all about the historical views and the ways of processing inequality that allowed the development of the concept of fragmentation.
\end{abstract}

Keywords: Fragmentation, Argentinean educational system, Educational inequality 


\section{Introducción}

Como fenómeno socio-educativo, la fragmentación es reciente en términos históricos. En Argentina se la identifica luego de las transformaciones que tuvo el sistema educativo a partir primero de la década de 1970 y luego en la década de 1990 en un contexto de redefinición del rol del Estado (FELDFEBER, 2000). Algunos de esos cambios, compartidos entre otros países de América Latina (KRAWCZYK; VIEIRA, 2008), incluían según Tiramonti la financiación del sector educativo privado en desmedro del público, la "provincialización" de la educación (es decir, el traspaso del financiamiento de la educación desde la nación hacia las provincias) y la generación de instituciones estatales cuyos roles fuesen la evaluación de resultados educativos y la rendición de cuentas con énfasis en la eficiencia del sistema, aunque también hubo un aumento del porcentaje de estudiantes en el nivel secundario (TIRAMONTI, 2001).

Junto con la caracterización de estas políticas y transformaciones en el sistema en ese período, este artículo interesa dar cuenta de cómo estas transformaciones dieron lugar a nuevas formas en que se expresó la fragmentación en la esfera de lo educativo. El análisis de la desigualdad educativa en relación a dichas políticas públicas da cuenta no sólo de cómo ésta se acrecentó, a pesar del aumento del acceso, sino que también se expresó en otras dimensiones de lo social.

Para ello, el presente artículo se organiza de la siguiente manera: luego de la introducción, un segundo apartado describe brevemente el sistema educativo argentino hasta la década de 1990 y presenta las principales transformaciones en términos de política educativa en las décadas de 1970 y 1990, poniendo énfasis en las rupturas.

El análisis histórico presente en el segundo apartado y el énfasis en las transformaciones del sistema permite dar cuenta los cambios en el modo en que se manifiesta la fragmentación educativa. Las consecuencias principales y los debates actuales en materia de desigualdad son expuestas en un cuarto apartado, el cual focaliza en cómo esa reconfiguración de lo educativo modificó asimismo los modos en que ésta se expresa. Finalmente, los apartados finales presentan las conclusiones del análisis.

El presente artículo da cuenta entonces qué condiciones permitieron la emergencia de los estudios sobre la fragmentación educativa en Argentina, cuáles fueron sus primeros referentes y que especificidades encontraron en relación al sistema educativo argentino, marcando semejanzas y diferencias. 


\section{Transformaciones del sistema: 1970 y 1990}

Desde mediados del siglo XIX, el sistema educativo argentino se estableció apuntando a una educación masiva y obligatoria bajo el interés principal de generar un ciudadano que incorporase una idea de nación argentina y su idioma. El ethos de la escuela se configuró a partir de una inclusión homogeneizante de todos sus habitantes sobre la base de una formación principalmente humanística (PUIGGRÓS, 2002) que fue promovida desde el Estado nacional y plasmada finalmente en la Ley de Educación Común №1420 del año 1884, que estableció su carácter obligatorio, estatal, laico y gradual para el nivel primario.

Si bien el texto constitucional de la época delegaba la responsabilidad a las provincias de proveer la educación elemental, fue el Estado nacional el actor político que financió y llevó a cabo la expansión del sistema en los territorios provinciales. Esto da cuenta del centralismo imperante en la época.

A lo largo del siglo XX, la expansión educativa y universalización del acceso comenzó en el nivel secundario. Dicha expansión se produjo en un momento inicial, durante el primer período de gobierno del peronismo (1946-1955), en gran parte con la expansión de las escuelas técnicas, y una expansión en las décadas de 1960 y 70 en particular en las escuelas con orientación comercial. A partir de entonces, el nivel secundario tuvo un crecimiento sostenido hasta la última dictadura militar (1976-1983), tal como se muestra en el Cuadro 1 realizado por la Dirección Nacional de Información y Evaluación de la Calidad Educativa (DINIECE), y luego tuvo una segunda etapa de expansión a partir de la década de 1990.

Cuadro 1: Evolución de los alumnos matriculados en la educación secundaria. Total del país, 1900-2006

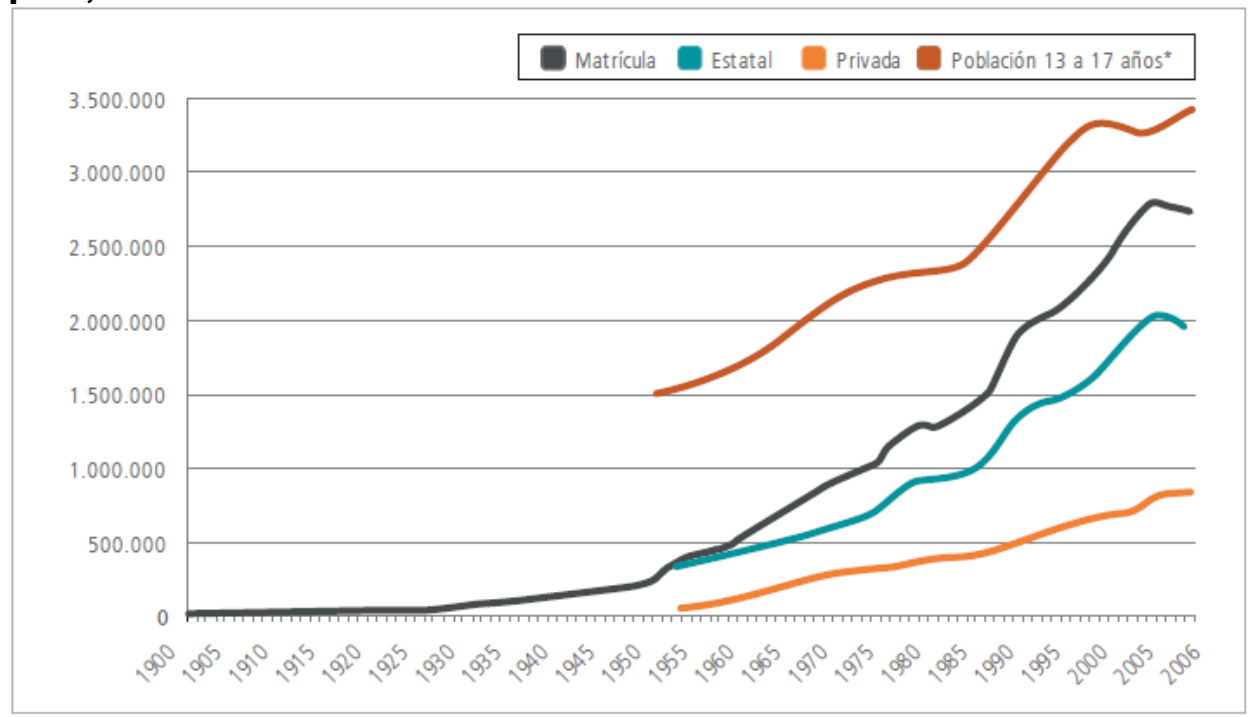

Fuente: DiNIECE (2007, p. 8) 
Investigaciones tales como la realizada por Filmus, Kaplan, Miranda y Moragues (2001) explican que la evolución y desarrollo del sistema educativo, particularmente en el nivel secundario, guardaba una íntima relación con los modelos productivos del país en cada momento histórico. En ese sentido, destacan que hasta entrada la década de 1930, la escolaridad en ese nivel estaba, por un lado, muy vinculada con la instrucción de élites en la modalidad de bachiller y por el otro lado con la formación docente. Hasta ese momento, era un nivel abiertamente selectivo, con examen de ingreso, y que experimentaba un bajo crecimiento tal como se evidencia en el Cuadro 1 de la Diniece.

A partir de 1946, en un contexto caracterizado por un mayor proteccionismo económico a nivel global, una industrialización por sustitución de importaciones en Argentina y con un proceso político que incluyo cierta democratización de la estructura social, comenzó un crecimiento sostenido de la matrícula del nivel secundario, sustentado sobre todo en la modalidad técnica y en menor medida por la modalidad comercial (GALLART, 1984), tal como se puede observar en los cuadros 2 y 3 :

Cuadro 2. Matrícula por modalidades en la enseñanza media (1900-1990)

\begin{tabular}{|l|l|l|l|l|l|l|}
\hline & Bachiller & Normal & Comercial & Técnica & Otras & Total \\
\hline 1900 & 3231 & 2185 & 1054 & 84 & 181 & 6735 \\
\hline 1910 & 7003 & 5435 & 2224 & 830 & 3162 & 17655 \\
\hline 1920 & 18281 & 16466 & 4946 & 2264 & 5932 & 47889 \\
\hline 1930 & 31035 & 23453 & 8714 & 6286 & 12576 & 82064 \\
\hline 1940 & 46464 & 45394 & 18863 & 16532 & 23271 & 150364 \\
\hline 1950 & 75915 & 62477 & 52148 & 69319 & 57692 & 317551 \\
\hline 1960 & 150718 & 138161 & 106317 & 91800 & 73965 & 560961 \\
\hline 1970 & 211273 & 192251 & 250888 & 138639 & 29183 & 1288642 \\
\hline 1980 & 510080 & 0 & 434250 & 315129 & 29183 & 1288642 \\
\hline 1990 & 770615 & 0 & 625830 & 413264 & 49616 & 1859325 \\
\hline
\end{tabular}

Fuente: Bonantini, C. (2000). Educación y Sociedad. Análisis histórico estructural de la escuela media argentina. Tomo II (1945-1983). Rosario: UNR editora. En base a: Ministerio de Educación y Justicia. Departamento de Estadísticas.

Cuadro 3. Matrícula por modalidades en la enseñanza media en porcentajes (1900-1990)

\begin{tabular}{|l|l|l|l|l|l|l|}
\hline & Bachiller & Normal & Comercial & Técnica & Otras & Total \\
\hline 1900 & $48,0 \%$ & $32,4 \%$ & $15,6 \%$ & $1,2 \%$ & $2,7 \%$ & $100,0 \%$ \\
\hline 1910 & $39,7 \%$ & $30,8 \%$ & $12,6 \%$ & $4,7 \%$ & $17,9 \%$ & $100,0 \%$ \\
\hline 1920 & $38,2 \%$ & $34,4 \%$ & $10,3 \%$ & $4,7 \%$ & $12,4 \%$ & $100,0 \%$ \\
\hline 1930 & $37,8 \%$ & $28,6 \%$ & $10,6 \%$ & $7,7 \%$ & $15,3 \%$ & $100,0 \%$ \\
\hline 1940 & $30,9 \%$ & $30,2 \%$ & $12,5 \%$ & $11,0 \%$ & $15,5 \%$ & $100,0 \%$ \\
\hline
\end{tabular}




\begin{tabular}{|l|l|l|l|l|l|l|}
1950 & $23,9 \%$ & $19,7 \%$ & $16,4 \%$ & $21,8 \%$ & $18,2 \%$ & $100,0 \%$ \\
\hline 1960 & $26,9 \%$ & $24,6 \%$ & $19,0 \%$ & $16,4 \%$ & $13,2 \%$ & $100,0 \%$ \\
\hline 1970 & $16,4 \%$ & $14,9 \%$ & $19,5 \%$ & $10,8 \%$ & $2,3 \%$ & $100,0 \%$ \\
\hline 1980 & $39,6 \%$ & $0,0 \%$ & $33,7 \%$ & $24,5 \%$ & $2,3 \%$ & $100,0 \%$ \\
\hline 1990 & $41,4 \%$ & $0,0 \%$ & $33,7 \%$ & $22,2 \%$ & $2,7 \%$ & $100,0 \%$ \\
\hline
\end{tabular}

Fuente: Bonantini, C. (2000). Educación y Sociedad. Análisis histórico estructural de la escuela media argentina. Tomo II (1945-1983). Rosario: UNR editora. En base a: Ministerio de Educación y Justicia. Departamento de Estadísticas.

La hipótesis sugerida por Filmus, Kaplan, Miranda y Moragues (2001) de que el crecimiento de determinadas modalidades de la enseñanza secundaria en las décadas de 1950 y 1970 estuvo ligada principalmente a las modificaciones en la estructura económica y productiva del país en los distintos momentos históricos explica algunas de las transformaciones en el sistema, pero hay que considerar también las reformas que se dieron en el orden de las políticas públicas.

A partir de la década de 1970 se realizaron varios cambios en la estructura del sistema educativo. Dicha década estuvo marcada por ser el primer proceso de descentralización del sistema educativo argentino (siendo el segundo llevado a cabo en la década de 1990). En 1968 se sancionó la ley No. 17.878 la cual enunciaba la transferencia de los establecimientos de enseñanza nacionales de nivel primario a las provincias. Ese traspaso incluía tanto los establecimientos, inmuebles y mobiliarios, como también los docentes, alumnos y personal en general.

Otro aspecto a destacar, tal como se puede observar en los cuadros 2 y 3 , es la eliminación a lo largo de la década de 1970 de la modalidad "normal", en la cual se realizaba la formación docente de nivel primario. En 1968 se cerraron las inscripciones a las escuelas normales (lo que da cuenta de la caída en el porcentaje de estudiantes de un $24 \%$ a un $14 \%$, particularmente por la falta de nuevos inscriptos), y en 1971 se instauró una nueva modalidad de formación docente caracterizada como "terciaria" (proceso que fue conocido como "terciarización" de la formación docente).

Asimismo, en esos años se produce una serie de cambios en el modelo de producción económica a nivel global que también tienen impacto en el país: durante la segunda postguerra se amplían las capas medias las cuales buscan cada vez más credenciales educativas. Segundo, la matriz productiva se modifica enormemente en particular luego de la crisis del petróleo lo cual produce nuevas pugnas y estrategias por el ingreso al mercado de trabajo que se reconfigura significativamente. $\mathrm{Y}$ finalmente, se expande de manera acelerada el circuito privado de educación el cual 
en un primer momento estaba restringido eminentemente a las comunidades nacionales o religiosas, pero a partir de entonces comienza a incorporar los sectores medios-altos de la población como signo de distinción.

El proceso de "provincialización" de la escolaridad primaria, la "terciarización" de la formación docente, y la privatización del sistema educativo (atendiendo particularmente a los estratos más altos de la sociedad) dieron lugar a una descentralización del rol del estado nacional en el sistema educativo.

Autores como Tiramonti y De Luca (2008) destacan que el proceso de descentralización de la década de 1970 tuvo dos dimensiones: "por un lado, mejorar la adaptabilidad del sistema a las necesidades de cada región. Pero, por el otro, se instauraba un mecanismo según el cual la escuela iba perdiendo su dimensión común, diversificándose distintos tipos de escolaridad" (2008, p. 382). Sin olvidar entonces que "lo común" en el sistema educativo argentino hasta la década de 1970 implicó una homogeneización con fuertes rasgos restrictivos y autoritarios, hay que destacar que el modo en que se produjo la diversificación del sistema contribuyó a profundizar la desigualdad al interior de lo que el sistema educativo ofrecía. Este aspecto es central para comprender cómo se analiza la desigualdad contemporáneamente en Argentina.

Posteriormente, durante la década de 1990 hubo una segunda gran expansión del nivel medio luego de un período de amesetamiento durante el gobierno de la última dictadura militar (1976-1983). Varios factores contribuyen a explicar este proceso expansivo en esa década. Por un lado, a partir de 1984 (con la recuperación de la democracia) se suprimen los exámenes de ingreso al nivel secundario común (y solo en los casos en que la demanda superara la oferta de un establecimiento, se asignarían vacantes por sorteo). También se modificó el régimen de sanciones.

Pero por otro lado, el crecimiento del desempleo durante la segunda mitad de la década de 1970 tuvo un impacto particular en los jóvenes que comenzaron a buscar mayores certificaciones educativas para acceder a un mercado laboral cada vez más reducido y competitivo (JACINTO, 2006).

En términos de modificaciones en la estructura del sistema educativo, en 1993 se sanciona la Ley Federal de Educación No 24.195 que propone realizar cambios estructurales sobre la base de la reforma realizada en España en la década de 1970 (Ley General de Educación de España No 14/1970). En términos generales, se expande la educación primaria a tres ciclos de tres años cada uno (el último de los cuales incorpora los dos primeros años de la secundaria), y los últimos tres años de la 
secundaria pasan a conformar un ciclo "polimodal" no obligatorio con cinco posibles orientaciones.

De acuerdo a las bases censales de Argentina, la tasa neta de escolarización en el nivel secundario pasó de 42,2\% en 1980 a 79,5\% en el año 2001. Sin embargo, dicho aumento de la matrícula estuvo acompañado por un incremento del índice de desgranamiento, es decir el abandono de los estudios en una misma cohorte de edad y una caída de la tasa de graduación, tal como se observa en el siguiente cuadro:

\section{Cuadro 4. Evolución de la tasa de graduación 1950-1992}

\begin{tabular}{|l|l|}
\hline Período & Tasa de graduación \\
\hline $1950-1955$ & $50,8 \%$ \\
\hline $1956-1960$ & $65,7 \%$ \\
\hline $1961-1965$ & $69,8 \%$ \\
\hline $1966-1970$ & $65,7 \%$ \\
\hline $1971-1975$ & $64,7 \%$ \\
\hline $1976-1980$ & $67,1 \%$ \\
\hline $1981-1985$ & $63,4 \%$ \\
\hline $1986-1990$ & $55,0 \%$ \\
\hline $1991-1992$ & $40,9 \%$ \\
\hline
\end{tabular}

Fuente: Giuliodori; Giuliodori; González, (2004). La deserción en el nivel medio de la educación en la República Argentina. Cálculo de tasas de abandono e identificación de algunos factores que se le asocian. Revista de Economía y Estadística, vol. XLII, Instituto de Economía y Finanzas, Universidad Nacional de Córdoba. En base a los datos de la Encuesta Permanente de hogares (EPH).

Otras consecuencias destacadas en relación a las modificaciones realizadas en la década de 1990 fueron, según Puiggrós (2002, p. 190):

1. Diversificación de la estructuración del sistema. A partir de entonces, coexistieron en Argentina sistemas educativos provinciales distintos: unos con primaria y secundaria de 6 años cada una, otras provincias con la modalidad tradicional, de 7 años en la primaria y 5 en la secundaria y finalmente otros sistemas con tres ciclos de 3 años cada uno para la primaria, y un cuarto ciclo correspondiente a la antigua secundaria ahora denominada "polimodal". Esto daba cuenta de una total desarticulación del sistema educativo nacional, dado que entre varios sistemas provinciales no había correspondencia.

2. Hubo lo que se denominó una "primarización" de los años iniciales del secundario, así como una convivencia de adolescentes con niños menores en el mismo establecimiento. Sumado a ello, estuvo el hecho de que no se prepararon a los directivos ni a los docentes de las instituciones de enseñanza primaria para atender a la población adolescente. 
3. Fueron cerradas diversas opciones de la enseñanza técnica, sin proponer una modalidad que la reemplace. Asimismo, hubo un deterioro de la educación de adultos, la educación especial y la modalidad artística.

4. Finalmente, Puiggrós destaca un fuerte recorte curricular a lo largo de la década de 1990.

Si bien es cierto que el recorte curricular implicó con el tiempo una mejora en la tasa de egreso, así como también la ampliación de la matrícula, el alza en esas variables no implicó una mejora en la calidad. Sumado a ello, la diversificación del sistema enunciada por Puiggrós se evidenciaba en la diversidad de estructuras, pero también en la diversidad de modalidades educativas, de planes curriculares provinciales y de programas tendientes a la búsqueda de mejorar las estadísticas de terminalidad.

Todos estos aspectos dieron lugar a una diversificación al interior del sistema jerarquizada por calidad, en donde los sectores de nivel socio económico (NSE) más bajos eran canalizados hacia las instituciones de menor prestigio y calidad. Sobre este aspecto se refiere el siguiente apartado.

\section{Consecuencias de la reestructuración del sistema}

\subsection{Fragmentación en la organización del sistema}

Sumadas a las consecuencias enunciadas por Puiggrós, las modificaciones propuestas por la Ley Federal de Educación encontraron resistencias al interior de cada provincia. Como mencionamos, si bien históricamente el sistema educativo argentino se caracterizó por ser centralizado, la Constitución Nacional aseguraba para cada provincia la autonomía de la administración de la educación primaria siempre que no se contradiga con el texto constitucional.

Cuando se efectivizó la Ley Federal de Educación, no todas las provincias modificaron sus sistemas de acuerdo con dicha ley en virtud de la autonomía que les otorgaba la constitución. Así, por ejemplo, la Ciudad de Buenos Aires y la provincia de Río negro mantuvieron la estructura anterior de siete años de educación primaria y cinco de secundaria. En otros casos, algunas provincias realizaron un nuevo y propio sistema, como la provincia de Córdoba que contraria a la propuesta nacional de extender la primaria a nueve años, la acortó a seis e instituyó una secundaria también de seis años dividida en dos ciclos. 
Finalmente, incluso entre aquellas provincias que acompañaron la nueva legislación, los modos de aplicación fueron muy diversos debido a la dificultad de generar ese cambio. Por ejemplo, los edificios de educación primaria no estaban preparados (tanto en tamaño como en el tipo de mobiliario y material didáctico) para incorporar a adolescentes de 12 a 14 años de edad. Tampoco ni los docentes ni los directivos fueron preparados con anticipación para ese nuevo estudiante.

El resultado de estos cambios derivó, según investigaciones como las de Rivas (2004) o la de la Facultad Latino Americana de Ciencias Sociales (FLACSO, 2000), en que se generasen más de treinta estructuras organizacionales en todo el territorio nacional, dificultando la regulación del sistema y generando diferencias internas más grandes de las que ya había.

Comparativamente, mientras el modelo imperante hasta la década de 1990 tuvo al Estado Nacional como eje articulador, las transformaciones llevadas adelante en aquella época instituyeron modificaciones profundas en la distribución de responsabilidades desde el estado hacia las provincias.

\subsection{Fragmentación en el sujeto de la educación}

Uno de los aspectos más destacables es el reconocimiento por parte del estado del abandono de la pretensión universalista en educación que caracterizó tanto al Estado Liberal de finales de siglo XIX y principios del XX, como al Estado de Bienestar de post-guerra. El encumbramiento de las políticas focalizadas supuso también la aceptación, en el marco de esas políticas, de una desigualdad social regulada por el mercado que no era cuestionada.

Este modelo de políticas focalizadas, conforma también una diversidad de sujetos hacia los cuales se implementaban esas políticas. El sujeto de la educación dejaba de ser el ciudadano moderno como sujeto de derecho, para abarcar una multiplicidad de sujetos que distintas investigaciones caracterizaron como de subjetividades tuteladas (DUSCHATZKY, 1999; DUSSEL; TIRAMONTI; BIRGIN, 1998).

\subsection{Continuidades post-neoliberalismo}

Otro aspecto destacable se presentó en la contradicción entre la expansión de los derechos en el plano de los sistemas normativos al mismo tiempo que se empobrecieron los recursos sociales (públicos y privados) que se requieren para la efectiva realización de esos derechos. En otras palabras, el alcance de las 
transformaciones que se realizaron no vino acompañada de un financiamiento acorde, sobre todo en términos de capacitación docente (que en muchos casos se dejó librada a la oferta privada de formación docente) ni en infraestructura, pero sí hubo un crecimiento importante de la matrícula.

Luego de la crisis económica, social y política en Argentina en diciembre de 2001, la cual culminó con la renuncia del entonces presidente de la nación, se inicia un período de crisis institucional hasta el año 2003, momento a partir del cual comienza a recuperarse la economía y se estabiliza la situación social.

A partir del año 2003, se observa un fuerte crecimiento de la inversión estatal en materia educativa, que lleva a un aumento del $99 \%$ de la inversión por alumno estatal en pesos constantes entre los años 1998 y 2015, tal como se muestra en el cuadro 5:

\section{Evolución de la inversión educativa como porcentaje del PIB y del gasto por alumno estatal, en pesos (1999-2015)}

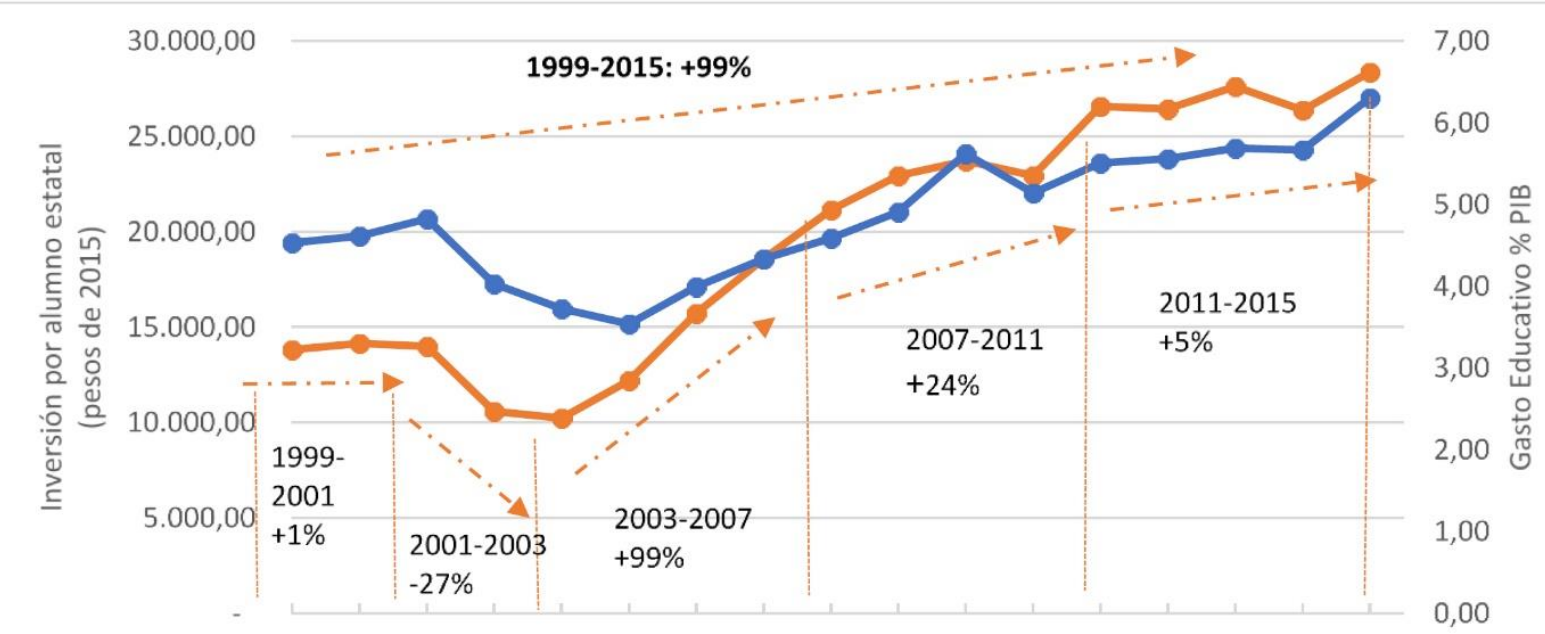

19992000200120022003200420052006200720082009201020112012201320142015

—Inversion por alumno estatal (en pesos de 2015) — - Gasto educativo \% PIB

Fuente: Cippec en base a Cgecse/DNPE/SlyCE/MEN, Secretaría de Política Económica, Ministerio de Hacienda e Indec (RIVAS; DBORKIN, 2018, p.13)

Sin embargo, autores como Kessler (2014) dan cuenta de que el aumento del presupuesto en educación en el decenio 2003-2013 no significó una disminución de la desigualdad educativa. Es decir: hubo un proceso de expansión tanto de la cobertura educativa como del presupuesto en educación, pero perduraron desigualdades vinculadas con la calidad, la cantidad de días y horas de clase, en los sueldos docentes y en el presupuesto que cada provincia destina a las políticas educativas. A este proceso, Kessler lo denominó como de "tendencias contrapuestas".

Otros autores, como Redondo (2009) señalan que además de las inequidades preexistentes como las recién mencionadas, en parte producto de la fragmentación del 
sistema, termina pesando también el proceso de selección de los establecimientos que realizan padres y alumnos y que consolidó una distribución segmentada de la población en edad escolar.

Resumidamente, las transformaciones suscitadas particularmente en las décadas de 1970 y de 1990 dieron lugar a una nueva configuración fragmentada del sistema que, en palabras de Tiramonti, "no es otra cosa que una transformación de los modos en que el sistema educativo procesa la desigualdad social" (TIRAMONTI, 2005, p.69-70). Para la autora, la desigualdad educativa no podía analizarse únicamente a partir de las variables clásicas como la exclusión o la deserción escolar, "sino que aquellos que asistían a la escuela eran incorporados a circuitos diferenciados por la calidad de los aprendizajes que allí se producían" (2005, p.70), sin por ello dejar de lado que las diferencias de calidad tenían un vínculo directo con el origen social del estudiantado. Lo que hasta la década de 1990 nombrábamos como sistema hoy no es otra cosa que un agregado institucional fragmentado.

\section{Los modos de la fragmentación}

Un aspecto crucial en el análisis de la fragmentación es tener en cuenta que ésta no solamente implica una diferenciación interna del sistema por tipos de instituciones, sino que afecta diversas variables.

Autores como Saraví (2015) enuncian como aspecto más destacable el fenómeno social que combina la desigualdad con la exclusión, conceptualizado como proceso de fragmentación y que en palabras de dicho autor "consiste en exclusiones recíprocas e inclusiones desiguales" (2015, p.21). Así, según el autor, "la fragmentación no es solamente el resultado exclusivo de la desigualdad económica (aunque ésta sea determinante en última instancia), sino que involucra además dimensiones culturales, sociales y subjetivas" (SARAVÍ, 2015, p.21).

En este sentido, según los análisis sobre la fragmentación, la profundización y persistencia de la desigualdad objetiva no es suficiente para poder explicar la evolución de la desigualdad: el punto es que la fragmentación en el ámbito educativo supone un distanciamiento social entre los espacios escolares y que tiene como consecuencia la emergencia de fronteras socioculturales que producen un extrañamiento recíproco.

En el caso argentino, este distanciamiento tiene impacto en distintos aspectos: primero, afecta no sólo a los estudiantes, sino a los diversos actores del ámbito educativo, particularmente los docentes. En segundo lugar, interviene en cómo se 
configura la experiencia escolar por fuera de la escuela misma. Tercero, la fragmentación se evidencia también en la relación educación y mercado de trabajo. Y finalmente reconfigura lo público y lo privado en las experiencias contemporáneas.

En relación a los efectos de la fragmentación en el cuerpo de docentes se destacan estudios como los de Poliak (2004). A partir de un estudio realizado en la provincia de Buenos Aires, la autora destaca que las escuelas fueron instituyendo a lo largo del tiempo diferentes criterios para seleccionar profesores como para definir el perfil del "profesor deseado". Así, ser hombre o ser mujer, ser joven, soportar las condiciones de trabajo, adecuarse a la disciplina establecida, los códigos de vestimenta, el tipo de cursos de formación que tomó, tener sentido de pertenencia institucional o simplemente contar con antigüedad son algunos de los múltiples elementos que dan cuenta de la pertenencia a instituciones, las cuales atienden a sectores poblacionales diferentes también (POLIAK, 2004).

En una investigación más reciente, Poliak (2009) da cuenta de que las escuelas reclutan docentes en función de determinados perfiles y que ese reclutamiento entrecruza mecanismos formales con informales (2009, p. 284). Por ejemplo, la autora menciona el caso de una directora de una escuela de sectores bajos que fue a un acto público de concurso docente "para alertar a los aspirantes sobre las condiciones y la ubicación de la escuela y desalentar a quienes no estuvieran dispuestos a enseñar bajo las mismas, dando cuenta de un uso estratégico de la normativa vigente" (2009, p. 285).

El segundo aspecto mencionado refiere a la experiencia escolar por fuera de la institución escolar: el aumento de la tasa de inscripción, vino acompañado también de mayores tasas de abandono, sobreedad y, según Kessler, de una experiencia educativa de "baja intensidad" (2002). Kessler entiende por ello que la experiencia de aprendizaje se restringe en muchos sectores sociales a lo que ocurra dentro del edificio escolar, dado que los jóvenes no cuentan con apoyo de familiares, ni con espacios para dedicarse al estudio (ni en sus casas, ni tampoco espacios públicos cercanos que ofrezcan esa posibilidad, como bibliotecas públicas).

En este sentido, por ejemplo, las clásicas "tareas para el hogar" marcan un diferencial más entre los distintos estudiantes que asisten a una misma escuela, pero cuyas realidades son divergentes. En esos casos la vida exterior ocupa un lugar mayor en la escuela, por lo que la subjetividad de esos estudiantes se va construyendo muy tamizada por experiencias de clase particulares. 
El tercer aspecto mencionado es la desvalorización de las credenciales educativas en el mercado laboral reciente, lo cual muestra una transformación de la relación entre las instituciones modernas educativas y el mercado laboral.

En términos más generales, para el caso de Francia, François Dubet (2003) da cuenta de que la escuela republicana del siglo XIX y XX pudo sostener la promesa de inclusión sobre la base de un ideario meritocrático y excluyente, porque quienes terminaban fuera del sistema escolar tenían garantizada una inclusión subordinada en el mercado de trabajo. En otras palabras: los jóvenes conseguían algún empleo sin importar sus cualificaciones escolares (más allá de las diferencias jerárquicas y de salario entre dichos empleos). Estas características podrían asemejarse a la situación argentina de la primera mitad del siglo XX: la tasa de prácticamente pleno empleo en el marco de un estado de bienestar servía, también, como amortiguador social frente a las diferencias que se daban en la escuela.

Sin embargo, con las transformaciones de la década de 1970, que incluyeron la masificación de la escuela, pero también la contracción del mercado laboral, derivó en que los más descalificados desde el punto de vista de credenciales educativas tengan muchas más posibilidades de ser excluidos sociales en relación a lo que sucedía antes. En el mismo sentido, Kessler (2010) menciona que "las expectativas generadas por la educación y los logros laborales luego obtenidos eran parte de un esquema propio de una época donde las expectativas de estabilidad laboral y movilidad social eran diferentes a las actuales" (KESSLER, 2010, p. 62).

Estas trayectorias educativas diferenciadas contrastan fuertemente con la pretensión universalista y homogeneizadora de la escuela de principios de siglo XX y que implica una larga reconversión del lazo social y que modifican el rol público de la escuela.

En un contexto de fragmentación educativa, las posibilidades de encontrarse con otros diferentes se reducen; ciertos sectores de clases altas o medias-altas buscan actualmente una socialización educativa que siga la traza de la socialización familiar; en otros casos se realiza una búsqueda por conformar universos culturales similares, que involucran barreras materiales y simbólicas. Todos estos aspectos, que involucran desigualdades de clase, pero también estrategias de diferenciación entre sectores poblacionales y baja institucionalización por parte del estado que fragmentan el entramado social. 


\section{Cierre}

La fragmentación es más que una referencia a la diversidad de las experiencias educativas. La transmutación de la desigualdad hacia la fragmentación social ocurre silenciosamente: las diferencias económicas no desaparecen (incluso, en muchos casos se han incrementado), pero además la desigualdad se filtró en otros espacios de la vida social. La fragmentación social se refiere a un distanciamiento sociocultural en muchos casos asociado a la desigualdad socioeconómica o de clase. Las exclusiones recíprocas en el espacio escolar contribuyen a la reproducción de la desigualdad económica pero también al distanciamiento social y cultural y, principalmente, al debilitamiento de la cohesión social.

La fragmentación en mundos aislados que se excluyen recíprocamente contribuye a la pérdida del reconocimiento del "otro" como miembro de una misma comunidad, como alguien con el que existe un mínimo vínculo de empatía. Donde antes había experiencias compartidas entre distintos sectores sociales, hoy ya no las hay, y la importancia de éstas reside en que contribuyen y alimentan la solidaridad social.

En este contexto, la respuesta no es volver a una escuela decimonónica, muchas veces idealizada pero que daba cuenta de una homogeneización con fuertes rasgos restrictivos y autoritarios, sino en pensar nuevas formas de re-establecer la dimensión pública de lo común.

\section{Referencias bibliográficas}

AGUERRONDO, I.; PRADO, M. Los planes de estudio vigentes en el nivel medio de orden nacional. Buenos Aires: Ministerio de Cultura y Educación de la Nación, 1990.

BIRGIN, A.; DUSCHATZKY, S.; DUSSEL, I. Las instituciones de formación docente frente a la reforma: estrategias y configuraciones de la identidad. Propuesta Educativa, n.19, p. 24-35, 1998.

BONANTINI, C. Educación y sociedad. Análisis histórico estructural de la escuela media argentina. Rosario: UNR editora, 2000. Tomo II (1945-1983).

BRASLAVSKY, C. La discriminación educativa en la Argentina. Buenos Aires: Miño y Dávila, 1989.

DINIECE. La obligatoriedad de la educación secundaria en Argentina. Deudas pendientes y nuevos desafíos. Buenos Aires: Ministerio de Educación, 2007. Disponível em: <https://www.argentina.gob.ar/sites/default/files/debate_4.pdf>. Acesso em: 03 dic. 2019.

DUBET, F. A escola e a exclusão. Cadernos de Pesquisa, n. 119, p. 29-45, jul 2003. 
DUSCHATZKY, S. La escuela como frontera. Buenos Aires: Ed. Paidós, 1999.

DUSSEL, I.; TIRAMONTI, G.; BIRGIN, A. Programas y proyectos en las escuelas: los alcances de la reforma escolar. Contexto \& Educação, n. 40, p. 26-48, 1995.

FELDFEBER, M. Una transformación sin consenso: apuntes sobre la política educativa del gobierno de Menem. Versiones, n.11, p. 8-20, Buenos Aires 2000.

FILMUS, D. et al. Cada vez más necesaria, cada vez más insuficiente, la escuela media en épocas de globalización. Buenos Aires: Santillana, 2001.

FLACSO. Ministerio de Cultura y Educación de la Nación. Proyecto estado de situación de la implementación del tercer ciclo de EGB en seis jurisdicciones. Buenos Aires: Ministerio de Cultura y Educación de la Nación, 2000.

GALLART, M.A. La evolución de la educación secundaria 1916-1970: El crecimiento cuantitativo de la matrícula y su impacto en la fuerza de trabajo (segunda parte).

Revista CIAS, v. 33, n. 331, p. 4-20, 1984.

GESSAGHI, V. La educación de la clase alta argentina: entre la herencia y el mérito. Buenos Aires: Siglo XXI Editores, 2016.

GIULIODORI, R.; GIULIODORI, M.A; GONZÁLEZ, M. La deserción en el nivel medio de la educación en la República Argentina. Cálculo de tasas de abandono e identificación de algunos factores que se le asocian. Revista de Economía y Estadística, v. 42, n. 1, p. 71-92, 2004.

JACINTO, C. La escuela media: reflexiones sobre la agenda de inclusión con calidad. Buenos Aires: Fundación Santillana, 2006.

KESSLER, G. La experiencia escolar fragmentada. Estudiantes y docentes en la escuela media de Buenos Aires. Paris: UNESCO, 2002.

La disyunción educación-trabajo en el Mercosur. Coincidencias y diferencias entre jóvenes de Argentina, Brasil, Paraguay y Uruguay. Propuesta Educativa, n. 34, p. 53-64, 2010.

Controversias sobre la desigualdad. Argentina 2003-2013. Buenos Aires:

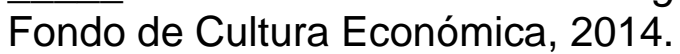

KRAWCZYK, N.; VIEIRA, V.L. A reforma educacional na América Latina nos anos 1990. Uma perspectiva histórico-sociológica. Revista Latinoamericana de Educación Comparada, año 1, n.1, p. 10-17,2010.

MAYER, L.; NÚÑEZ, P. Nuevas y viejas desigualdades en la experiencia educativa juvenil en América Latina. Temas, n.87-88, p.33-40, 2016.

POLIAK, N. Reconfiguraciones recientes en la educación media: escuelas y profesores en una geografía fragmentada. In: TIRAMONTI, G. (Comp.) La trama de la desigualdad educativa. Mutaciones recientes en la escuela media. Buenos Aires: Ed. Manantiales, 2004. p. 147-192.

Fragmentación educativa en el campo docente: acerca de criterios de selección de profesores en escuelas medias de la Ciudad de Buenos Aires. Espacios en Blanco. Revista de Educación, v. 19, p. 267-298, jun 2009.

PUIGGRÓS, A. Qué pasó en la educación argentina. Breve historia desde la conquista hasta el presente. Buenos Aires: Galerna, 2002.

REDONDO, J. La educación chilena en una encrucijada histórica. Diversia. Educación y sociedad, v. 1, n.1, p.13-39, 2009.

Revista Educação Online, Rio de Janeiro, n. 35, set-dez 2020, p. 176-191 
RIVAS, A. Gobernar la educación. Estudio comparado sobre el poder y la educación en las provincias argentinas. Buenos Aires: Granica - Universidad de San Andrés, 2004.

RIVAS, A.; DBORKIN, D. ¿Qué cambió en el financiamiento educativo en Argentina? In: CENTRO DE IMPLEMENTACIÓN DE POLÍTICAS PÚBLICAS PARA LA EQUIDAD Y EL CRECIMIENTO - CIPPEC. Documento de Trabajo N¹62. Buenos Aires: CIPPEC, Programa de Educación, Área de Desarrollo Social, febrero 2018. Disponível em: <https://www.cippec.org/wp-content/uploads/2018/02/DT-162-EDUFinanciamiento-educativo_2018-01-VF-2.pdf>. Acesso em: 03 dic. 2019.

SARAVÍ, G. De la desigualdad a la fragmentación. In: . Juventudes fragmentadas. Socialización, clase y cultura en la construcción de la desigualdad. México: FLACSO, 2015. p. 25- 56.

TEDESCO, J.C. Educación y sociedad en la Argentina (1880-1945). Buenos Aires: Ediciones del Solar, 1986.

TILLY, C. La desigualdad persistente. Buenos Aires: Manantial, 2000.

TIRAMONTI, G. Modernización educativa de los noventa. ¿El fin de la ilusión emancipatoria? Buenos Aires: FLACSO, 2001.

. La educación argentina en el contexto de las transformaciones de los años 90. Pro-Posições, v. 16, n.3, (48), p. 53-74, set-dez 2005.

; DE LUCA, R. La descentralización antes de la descentralización: políticas educativas durante el gobierno de Onganía. Revista de la Escuela de Ciencias de la Educación, año 4, n. 3, p-375-389, 2008.

; BRASLAVSKY, C.; FILMUS, D. Las transformaciones de la Educación en 10 años de democracia. Buenos Aires: FLACSO; Tesis Norma, 1995. 